\title{
Identifying Land Usage from Aerial Image using Feature Fusion of Thepade's Sorted n-ary Block Truncation Coding and Bernsen Thresholding with Ensemble Methods
}

\author{
Sudeep D. Thepade, Piyush R. Chaudhari, Rik Das
}

\begin{abstract}
Automatic Land Usage Identification is one of the most demanded research areas in Remote Sensing. One of the primitive sources for Land Usage Identification is Aerial images. Automatic Land Usage Identification is implemented by exploring different feature extraction methods whereas, these features are categorized into local and global content description of image. Fusion of local and global features may be a potential approach for land usage identification. Accordingly, the major contribution of work presented here is fusion of global color features extracted using TSBTC n-ary method (applied on entire image) and local features extracted using Bernsen thresholding method applied on $3 * 3$ windows of image for land usage identification. Consideration of more than one machine learning classifiers as an ensemble has shown better results than that of individual machine learning classifiers. In proposed work here, Thepade's Sorted n-ary Block Truncation Coding (TSBTC n-ary) is explored in aerial image feature extraction with nine variations from TSBTC 2-ary till TSBTC 10-ary. The performance appraise of proposed Land Usage Identification technique is done using UC Merced Dataset having 2100 images categorized into 21 land usage types. In consideration performance measures like Accuracy, F Measure and Matthews Correlation Coefficient (MCC); the TSBTC 10-ary global features extraction method has given better land usage identification as compare to Bernsen thresholding local feature extraction method. The proposed method enhances the identification of land usage through feature level fusion of TSBTC 10-ary global features and Bernsen thresholding local features. Along with nine individual machine learning algorithms, ensembles of varied machine learning algorithms are used for further performance improvement of the proposed land usage identification technique.
\end{abstract}

Keywords: Aerial Image, Bernsen, Land Usage Identification, Fusion, Machine Learning Algorithms, Thepade Sorted Block Truncation Coding.

\section{INTRODUCTION}

In today's era of satellites, Drones and Unmanned Aerial Vehicle (UAV); the technology allows acquisition of aerial imagery with details about what is there on the earth surface. Knowing more meaningful information from these details would be an interesting affair.

Revised Manuscript Received on February 05, 2020.

* Correspondence Author

Dr. Sudeep D. Thepade*, Computer Engineering Department, Pimpri Chinchwad College of Engineering, Pune, India. Email: sudeepthepade@gmail.com

Piyush R. Chaudhari, Computer Engineering Department, Pimpri Chinchwad College of Engineering, Pune, India. Email: piyushrc26@gmail.com

Rik Das, Information Technology Department, Xavier Institute of Social Service, Ranchi, Jharkhand, India. Email: rikdas78@gmail.com

(C) The Authors. Published by Blue Eyes Intelligence Engineering and Sciences Publication (BEIESP). This is an open access article under the CC BY-NC-ND license (http://creativecommons.org/licenses/by-nc-nd/4.0/)
The advancements in machine learning may help for automatic detection of the types of land usage through such aerial images. The paper uses appropriate machine learning algorithms to understand the extracted features from aerial images to uncover the usages of land in aerial images. Along with individual machine learning algorithms, the ensembles are also being explored to get better performance. The paper is formatted in various sections here. Section I gives an introduction, Section II contains a brief literature survey. Section III contains model for proposed land usage identification technique. Section IV comprises of results and discussion obtained for the proposed technique and the conclusion is given in Section V.

\section{LITERATURE REVIEW}

A great number of research papers propose the use of deep convolutional neural network architecture model for land usage identification compared to the models using various machine learning algorithms. Deep learning feature extraction requires a huge amount of computational power also scarcity of labeled datasets makes them a bit unreliable for usage. Hence umpteen efforts are taken to explore combinations of machine learning algorithms to identify the meaningful data from the extracted features of the individual aerial images. 


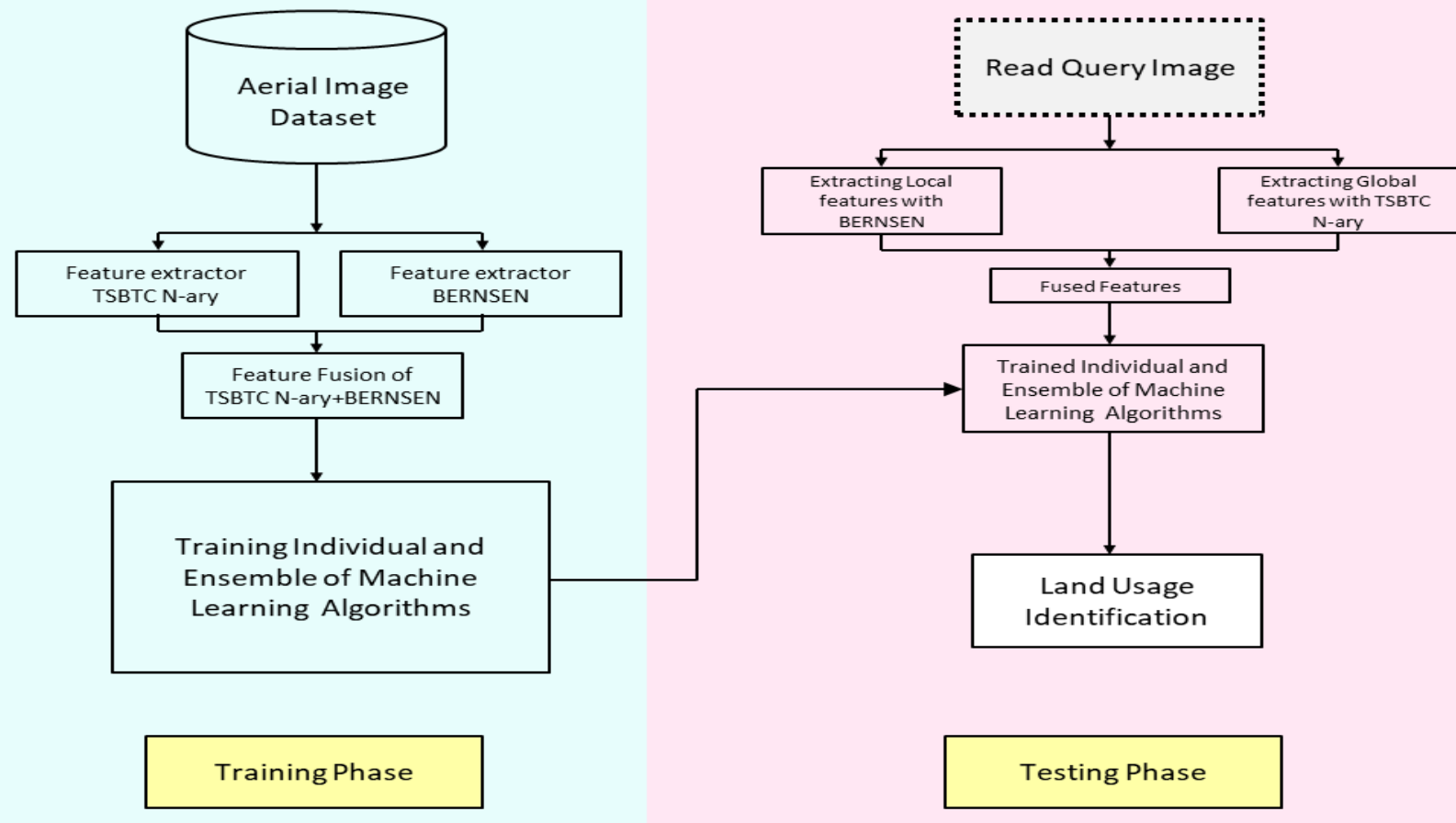

In [6] authors have proposed content-based remote sensing image retrieval using a fusion of color and texture features. Fused features obtained from color and texture are labeled using K-means clustering algorithm whereas to compare the similarity Manhattan distance is used. This work explored the wavelet transform for texture feature extraction which may not provide the required utmost potential performance for identifying land usage labels.

In [4] authors have proposed effective feature extraction and representation-based fusion strategy for land usage identification of provided aerial images. Local features are extracted using Bag of Visual Model (BoVM) and Spatial Pyramid Matching (SPM) and global feature extraction using Multiscale Completed Local Binary Patterns (MS-CLBP). Further Kernel Collaborative Representation-based Classification (KCRC) is used on extracted global and local features where the aerial images are annotated as per minimum approximation residual after the fusion step.

The method of Bag of Visuals Subgraphs (BoVSG) is proposed in [5] where segmentation of the image is done into superpixels containing only relevant information, such type of superpixels collected from each image clustered as

per there texture and color features and assigned with a land usage label. Superpixels belonging to the identical cluster should have the identical land usage label. This method takes less computational resources compared to deep learning methods. The authors in [13] proposed method generating four types of unsupervised features designed from four different CNN layers considering implementation of local binary pattern (LBP), gray-level co-occurrence (GLCM), maximal response 8 (MR8), and scale-invariant feature transform (SIFT) feature descriptors using collaborative affinity metric fusion to identify similarity in images.

Paper [14] proposes the framework named multi-feature joint sparse coding (MFJSC) having a spatial relation constraint classifier to superimpose spatial relation constraint. Limited capability of describing low level features is major drawback of [14] framework.

The main contribution of the work presented here is feature fusion based land usage identification using aerial images with help of global features extracted using Thepade's Sorted Block Truncation Coding (TSBTC n-ary) and local feature extracted using Bernsen thresholding method.

\section{PROPOSED AERIAL IMAGERY LAND USAGE IDENTIFICATION TECHNIQUE WITH FUSION OF GLOBAL TSBTC N-ARY FEATURES AND LOCAL BERNSEN THRESHOLDING METHOD}

The proposed land usage identification technique is architectured into two phases: Training phase and Testing Phase as shown in Fig 1. Training of individual and ensemble of machine learning algorithms is performed on the aerial images taken from the dataset using extraction of features from each image using "Thepade Sorted n-ary Block Truncation Coding" method as well as "Bernsen" Thresholding logic. Moreover, feature fusion of "TSBTC n-

ary" and "Bernsen" thresholding features is considered together in proposed method.

Further in Testing phase query aerial image is read whose features are extracted using considered global (TSBTC nary) and local (Bernsen thresholding) feature extraction methods which in-turn provided to trained machine learning algorithms or trained ensemble for land usage identification. The mathematical models of TSBTC n-ary and local feature extraction method using Bernsen thresholding are elaborated in subsections below. 
A. $\quad$ Thepade's Sorted n-ary Block Truncation Coding (TSBTC n-ary) [1] based Aerial Image Global Feature Extraction

Let the aerial image be 'AE' of size 'rxcx3', with three color planes as R, G and B. The feature vector of extracted using TSBTC n-ary may be considered as [TR1, TR2,.., TRn, TG1, TG2,.., TGn, TB1, TB2,.., TBn] . Here the TRi, TGi and $\mathrm{TBi}$ are indication the $\mathrm{i}$ 'th cluster centroids of individual color planes computed using TSBTC n-ary.

The TSBTC 2-ary can be elaborated using equations 1 to 6. Let sortR, sortG and sortB be the sorted representation single dimensional arrays of respective color planes $R, G$ and $\mathrm{B}$ of the aerial image.

$$
T R_{-} 1=2 / r x c \sum_{-}(p=1)^{\wedge}(r x c / 2) \operatorname{sortR}(p)
$$

$T R \_2=2 / r x c \sum_{-}(p=1+r x c / 2)^{\wedge} r x c \operatorname{sortR}(p)$

$$
T G \_1=2 / r x c \sum_{-}(p=1)^{\wedge}(r x c / 2) \operatorname{sort} G(p)
$$

$$
T G \_2=2 / r x c \sum_{-}(p=1+r x c / 2)^{\wedge} r x c \operatorname{sort} G(p)
$$

$$
T B \_1=2 / r x c \sum_{-}(p=1)^{\wedge}(r x c / 2) \operatorname{sortB}(p)
$$

$$
\begin{array}{r}
T B \_2=2 / r x c \sum_{-}(p \\
=1+r x c / 2)^{\wedge} r x c \operatorname{sortB}(p)
\end{array}
$$

Here in proposed aerial image land usage identification, in all ten variations of TSBTC n-ary are experimented as TSBTC-2ary, TSBTC-3ary, TSBTC-4ary, TSBTC-5ary, TSBTC-6ary, TSBTC-7ary, TSBTC-8ary, TSBTC-9ary and TSBTC-10ary.

\section{B. Bernsen Thresholding based Aerial Image Local Feature Extraction [2, 3]}

Here the Bernsen thresholding method is employed for extraction of local features of the aerial images. The Bernsen thresholding is implemented over the window size of $3 \times 3$ for each color plane of the aerial image.

Let the 'AE' be the aerial image of size ' $r x c x 3$ ', with three color planes as $\mathrm{R}, \mathrm{G}$ and $\mathrm{B}$. The feature vector extracted using Bernsen thresholding may be considered as [BTR1, BTR2, BTG1, BTG2, BTB1, BTB2]. The values of BTR1, BTR2, BTG1, BTG2, BTB1 and BTB2 are computed as per equations 16 to 21 .

For each local window ' $\mathrm{W}$ ' of size $3 \times 3$ of the respective color planes of aerial image 'AE' the local contrasts $\mathrm{TR}_{\mathrm{W}}$, TG $\mathrm{W}_{\mathrm{W}}$ and $\mathrm{TB}_{\mathrm{W}}$ could be found as given in equations 7, 8 and 9. Let the $W R_{\text {high }}$ and $W R_{\text {low }}$ be the highest and lowest intensity values of respective pixel window. Also let $\mathrm{CR}_{\mathrm{w}}$, $\mathrm{CG}_{\mathrm{w}}$ and $\mathrm{CB}_{\mathrm{w}}$ be the contrast values for respective color planes for the pixel window ' $\mathrm{W}$ ' as given in equations 10 , 11 and 12.

$$
\begin{aligned}
& \| T R \rrbracket_{-} W=\left(\llbracket W R \rrbracket_{-}\right. \text {high } \\
& +\varangle W R \nabla_{\text {_low }) / 2} \\
& \| T G \rrbracket_{-} W=\left(\varangle W G \rrbracket_{-}\right. \text {high } \\
& +\Delta W G \nabla_{\text {_low }) / 2} \\
& \| T B \rrbracket_{-} W=\left(\varangle W B \rrbracket_{-}\right. \text {high } \\
& +\| W B \rrbracket \text { _low) } / 2 \\
& \llbracket C R \rrbracket_{-} W=\left(\varangle W R \rrbracket_{-} \text {high }-\varangle W R \rrbracket_{-} \text {low }\right) \\
& \llbracket C G \rrbracket_{-} W=\left(\| W G \rrbracket_{-} \text {high }-\varangle W G \rrbracket_{\text {_low }}\right) \\
& \llbracket C B \rrbracket_{-} W=\left(\llbracket W B \rrbracket_{-} \text {high }-\varangle W B \rrbracket_{-} \text {low }\right)
\end{aligned}
$$

Then for the pixel window centered at $(\mathrm{x}, \mathrm{y})$ the respective color plane bitmaps BMR, BMG and BMB are generated as per equations 13,14 and 15.

These color plane bitmaps are used with the individual color planes to generate the feature vector elements as shown in equations 16 to 21 .

$$
\begin{aligned}
& B M R(x, y)=\left\{0, \mathbb{Z}\left[\text { if } \mathbb { U } \left(C R \rrbracket \_W\right.\right.\right. \\
& \left.<T R \rrbracket_{-} W\right) \& \& \quad \llbracket\left(T R \rrbracket_{-} W\right. \\
& \geq 128)] \text { or } \llbracket\left[i f \quad \llbracket \left(C R \rrbracket_{-} W\right.\right. \\
& \left.>T R \rrbracket \_W\right) \& \& \llbracket(R(x, y) \\
& \left.\left.\geq T R \rrbracket \_W\right)\right] 1 \text {, } \llbracket\left[i f \llbracket \left(C R \rrbracket \_W\right.\right. \\
& \left.<T R \rrbracket \_W\right) \& \& \llbracket\left(T R \rrbracket \_W\right. \\
& <128)] \text { or } \mathbb{Z}\left[\text { if } \llbracket \left(C R \rrbracket \_W\right.\right. \\
& \left.>T R \rrbracket \_W\right) \& \&(\llbracket R(x, y) \\
& <T R \rrbracket[W)] \\
& B M G(x, y)=\left\{0, \mathbb{Z}\left[\text { if } \mathbb { Z } \left(C G \rrbracket \_W\right.\right.\right. \\
& \left\langle T G \rrbracket \_W\right) \& \& \llbracket\left(T G \rrbracket \_W\right. \\
& \geq 128)] \text { or } \llbracket\left[i f \llbracket \left(C G \rrbracket \_W\right.\right. \\
& \left.>T G \rrbracket \_W\right) \& \& \llbracket(G(x, y) \\
& \left.\left.\geq T G \rrbracket_{-} W\right)\right] 1 \text {, } \llbracket\left[\text { if } \llbracket \left(C G \rrbracket \_W\right.\right. \\
& \left.<T G \rrbracket_{-} W\right) \& \& \llbracket(T G \rrbracket+W \\
& <128)] \text { or } \mathbb{[ i f} \llbracket\left(C G \rrbracket \_W\right. \\
& >T G \rrbracket+W) \& \&(\llbracket G(x, y) \\
& \left.\left.<T G \rrbracket_{-} W\right)\right] \\
& B M B(x, y)=\left\{0, \mathbb{U}\left[\text { if } \llbracket \left(C B \rrbracket \_W\right.\right.\right. \\
& \left.<T B \rrbracket_{-} W\right) \& \& \llbracket\left(T B \rrbracket \_W\right. \\
& \geq 128)] \text { or } \llbracket[i f \llbracket(C B \rrbracket+W \\
& \left.>T B \rrbracket \_W\right) \& \& \llbracket((B(x, y) \\
& \left.\left.\geq T B \rrbracket \_W\right)\right] 1 \text {, } \llbracket\left[\text { if } \llbracket \left(C B \rrbracket \_W\right.\right. \\
& \left.<T B \rrbracket_{-} W\right) \& \& \llbracket\left(T B \rrbracket_{-} W\right. \\
& <128)] \text { or } \mathbb{Q}\left[\text { if } \llbracket \left(C B \rrbracket \_W\right.\right. \\
& \left.>T B \rrbracket \_W\right) \& \&(\llbracket G(x, y) \\
& \left.\left.<T B \rrbracket \_W\right)\right]
\end{aligned}
$$

$$
\begin{aligned}
& B T R 1=1 /\left(\sum_{-}(x=1)^{\wedge} r \sum_{-}(y=1)^{\wedge} c[B M R(x, y)]\right) \sum_{-}(x \\
& =1)^{\wedge} r \sum_{-}(y \\
& =1)^{\wedge} c[B M R(x, y) * R(x, y)] \\
& B T R 2=1 /\left(\sum_{-}(x=1)^{\wedge} r \sum_{-}(y\right. \\
& \left.=1)^{\wedge} c[1-B M R(x, y)]\right) \sum_{-}(x \\
& =1)^{\wedge} r \sum_{-}(y \\
& =1)^{\wedge} c\{[1-B M R(x, y)] * R(x, y)\} \\
& B T G 1=1 /\left(\sum_{-}(x=1)^{\wedge} r \sum_{-}(y=1)^{\wedge} c[B M G(x, y)]\right) \sum_{-}(x \\
& =1)^{\wedge} r \sum_{-}(y \\
& =1)^{\wedge} c[B M G(x, y) * G(x, y)] \\
& B T G 2=1 /\left(\sum_{-}(x=1)^{\wedge} r \sum_{-}(y\right. \\
& \left.=1)^{\wedge} c[1-B M G(x, y)]\right) \sum_{-}(x \\
& =1)^{\wedge} r \sum_{-}(y \\
& =1)^{\wedge} c\{[1-B M G(x, y)] * G(x, y)\} \\
& B T B 1=1 /\left(\sum_{-}(x=1)^{\wedge} r \sum_{-}(y=1)^{\wedge} c[B M B(x, y)]\right) \sum-(x) \\
& =1)^{\wedge} r \sum_{-}(y \\
& =1)^{\wedge} c[B M B(x, y) * B(x, y)] \\
& B T B 2=1 /\left(\sum_{-}(x=1)^{\wedge} r \sum_{-}(y\right. \\
& \left.=1)^{\wedge} c[1-B M B(x, y)]\right) \sum_{-}(x \\
& =1)^{\wedge} r \sum_{-}(y \\
& =1)^{\wedge} c\{[1-B M B(x, y)] * B(x, y)\}
\end{aligned}
$$




\section{Identifying Land Usage from Aerial Image using Feature Fusion of Thepade's Sorted n-ary Block Truncation Coding and Bernsen Thresholding with Ensemble Methods}

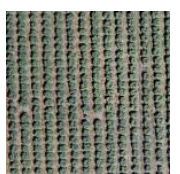

(a)

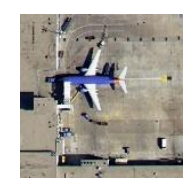

(b)

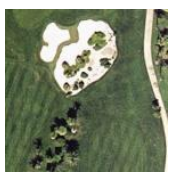

(j)

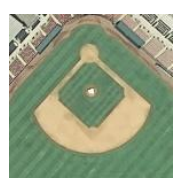

(c)

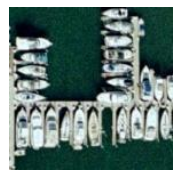

$(\mathrm{k})$

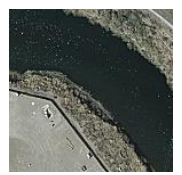

(q)

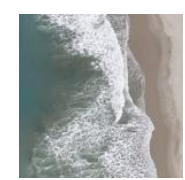

(d)

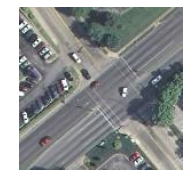

(l)

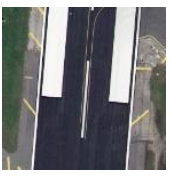

(r)

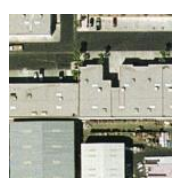

(e)

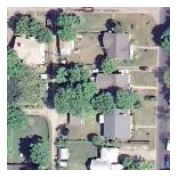

(m)

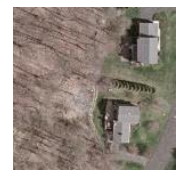

(s)

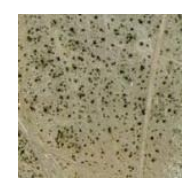

(f)

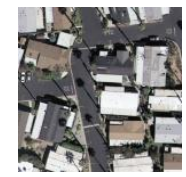

(n)

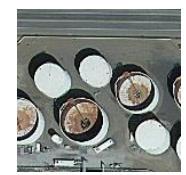

(t)

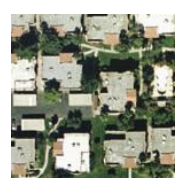

(g)

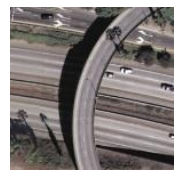

(o)

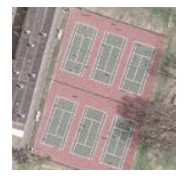

(u)

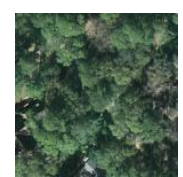

(h)

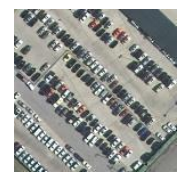

(p)

(a). agricultural, (b). airplane, (c). baseball diamond, (d). beach, (e). buildings, (f). chaparral, (g). dense residential, (h). forest, (i). freeway, (j). golf course, (k). harbor, (l). intersection, (m). medium residential, (n). mobile home park, (o). overpass, (p). parking lot, (q). river, (r). runway, (s). sparse residential, (t). storage tanks, (u). tennis court. Fig. 2 sample image from UC Merced Land Usage Database [12]

\section{RESULTS AND DISCUSSION}

The proposed land usage identification technique is evaluated on UC merced land usage dataset. The 228 variations of proposed technique are explored with set of nine machine learning algorithms and three ensemble combinations, nine variations of using TSBTC n-ary global feature level extraction method, Bernsen local feature level extraction method and nine variations of feature level fusion of global and local features. Fig. 2 is showing the sample images (one from each of land usage categories) from UC merced land usage image dataset [12] for 21 categories.

Performance based on percentage accuracy for proposed land usage identification technique using different variations of TSBTC n-ary global level feature extraction method is given in Fig. 3 for examined machine learning algorithms and ensembles. Here gradual increase in performance along with increasing level of TSBTC n-ary for each individual machine learning algorithm is observed. A fair bit increase in percentage accuracy is observed initially from TSBTC 2ary to TSBTC 8-ary. From TSBTC 8-ary to TSBTC 10-ary minimal increment in percentage accuracy is observed, whereas comprehensive best performance based on percentage accuracy is given by TSBTC 10-ary and ensemble of "IB1+Random Forest+ Simple Logistic", "IB1+Random Forest+ KStar" and "IB1+Random Forest+ Simple Logistic+ SMO+ KStar".

The comparison of experimented nine machine learning algorithms (Bayes Network, Simple Logistic, Binary SMO, KStar, IB1, J48, Random Forest, Random Tree, REPTree) and three ensembles of "IB1+Random Forest+ Simple Logistic", "IB1+Random Forest+ KStar" and "IB1+Random Forest +Simple Logistic +SMO+ KStar" based on percentage accuracy is given in Fig. 4 for respective TSBTC n-ary global feature extraction method variations in proposed land usage identification technique shows that the ensembles of almost all variations of global feature level extraction TSBTC n-ary method perform better than individual machine learning algorithms. Best comprehensive result is given by ensemble: "IB1+Random Forest+ Simple Logistic+ SMO+ KStar" and next second best result given by ensemble: "IB1+Random Forest+ Simple Logistic". 


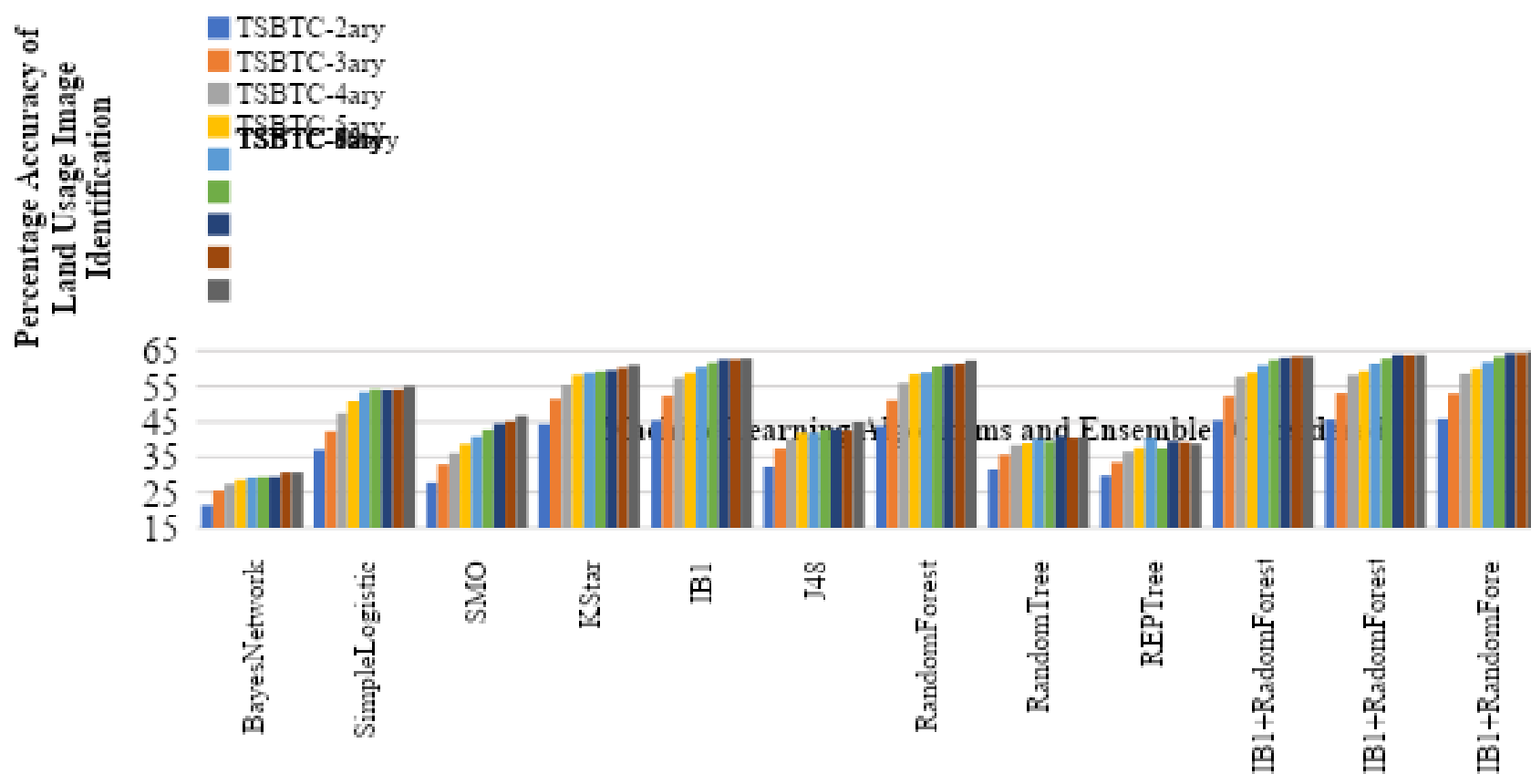

Fig. 3.Performance based on percentage accuracy of variations of TSBTC n-ary global features for respective machine learning algorithm in proposed land usage identification technique.

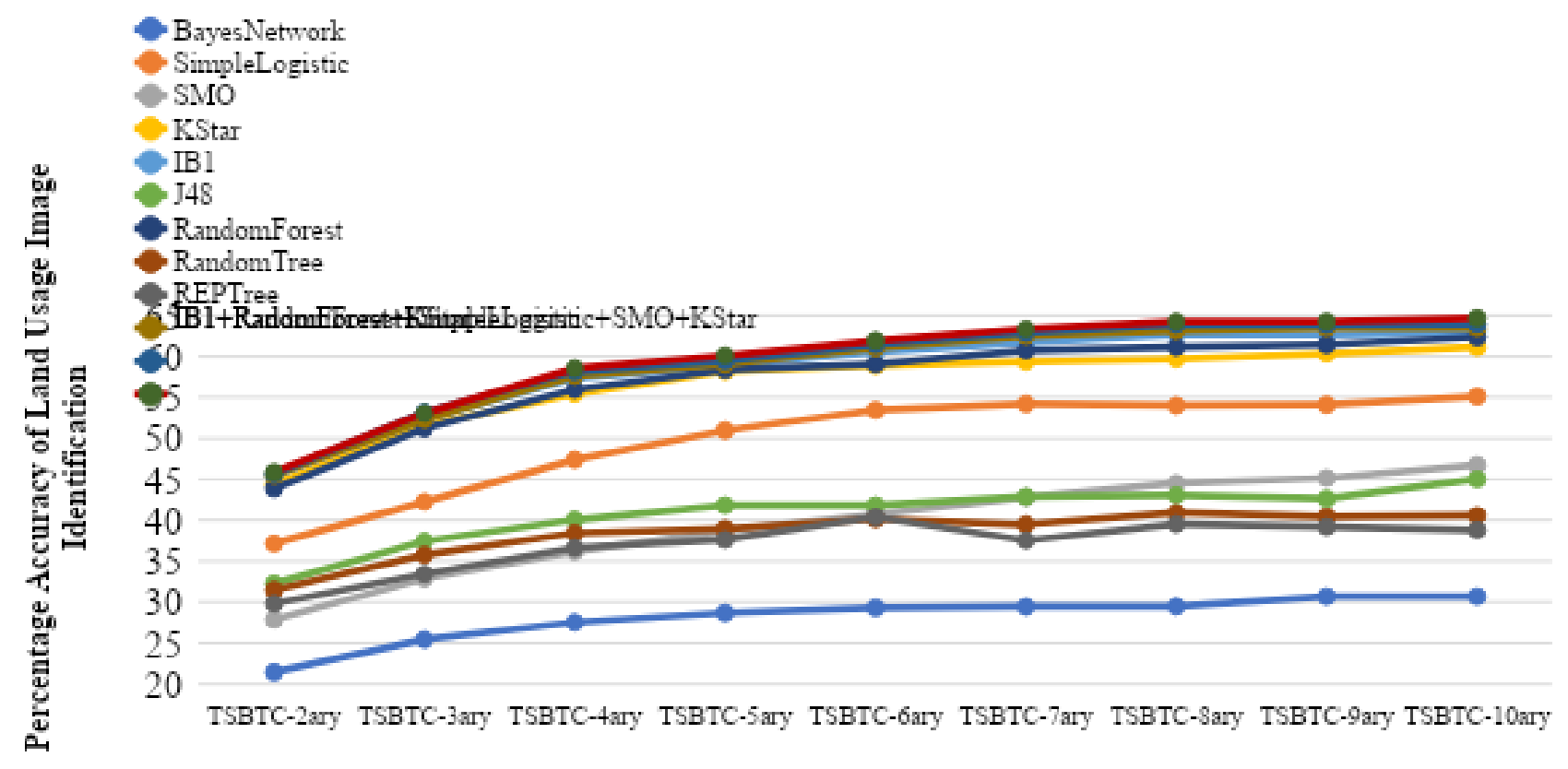

Respective Feature Vector Extraction Method Variations

Fig. 4.Performance based on percentage accuracy of examined machine learning algorithms and ensembles for respective TSBTC n-ary global feature vector extraction method variations in proposed land usage identification technique. 

Truncation Coding and Bernsen Thresholding with Ensemble Methods

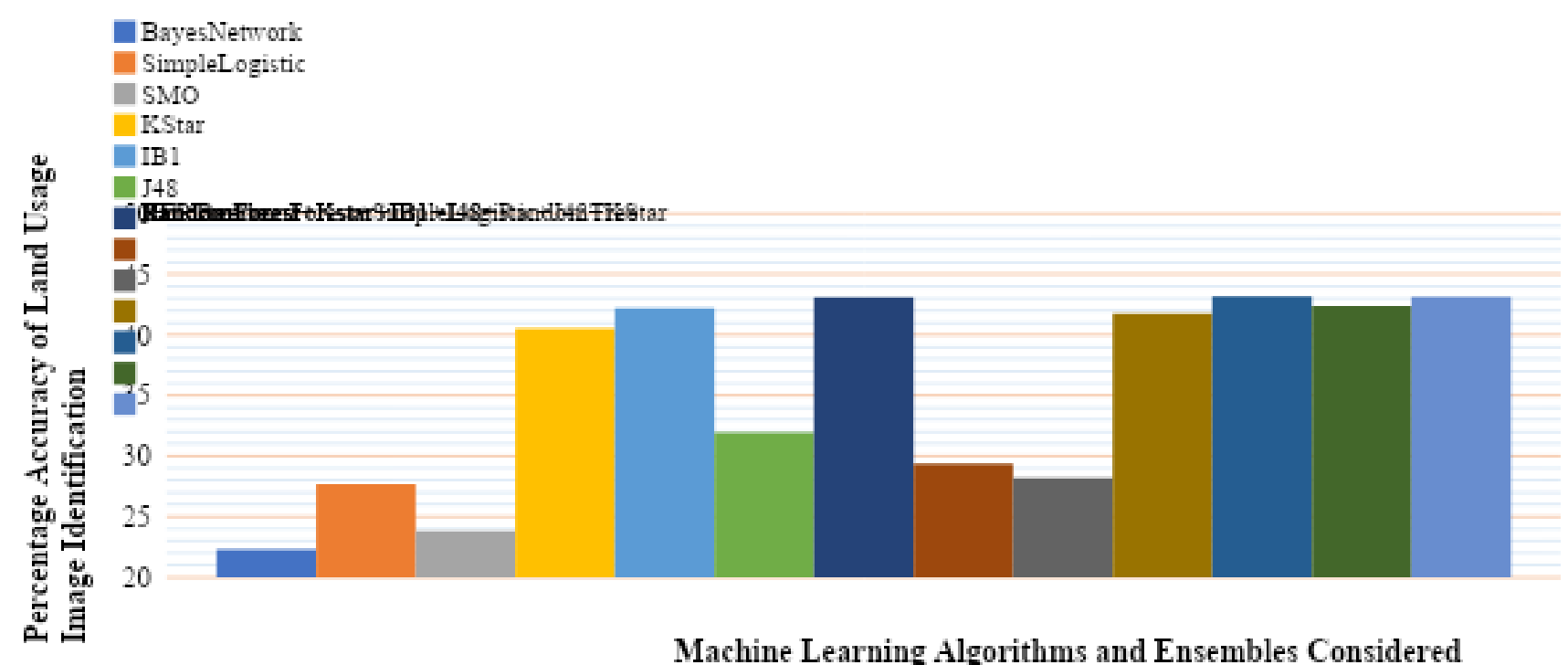

Fig. 5.Performance based on percentage Accuracy of examined machine learning algorithms and ensembles for the Bernsen thresholding based local feature extraction method in proposed land usage identification technique.

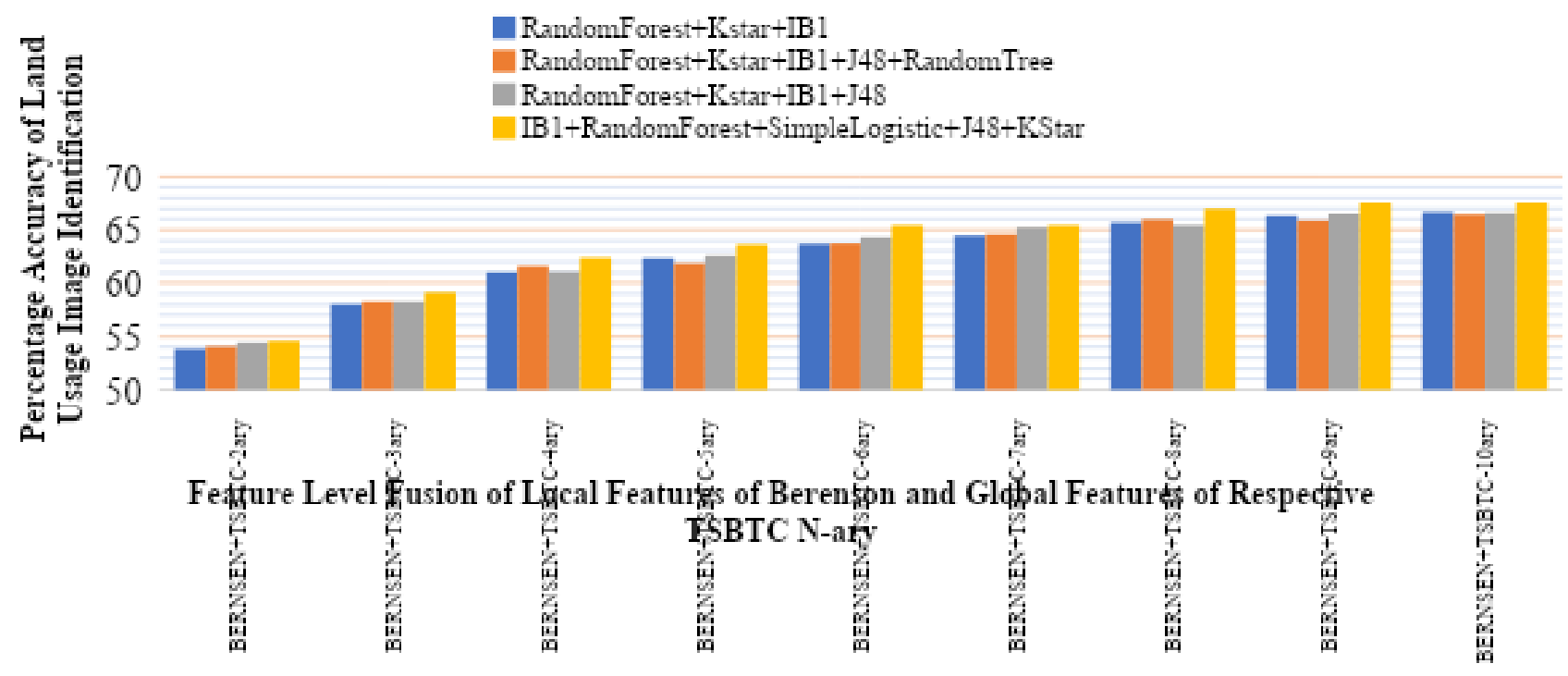

Fig. 6.Performance based on percentage Accuracy of examined ensembles of machine learning algorithms for respective feature level fusion combinations of local features of Bernsen and global features of respective TSBTC nary in proposed land usage identification technique.

Fig. 5 comprises of performance based on percentage Accuracy of examined individual machine learning algorithms and ensembles for Bernsen thresholding based local feature level vector extraction method in proposed land usage identification technique. As per the results, ensembles have the higher percentage accuracy of land usage identification in contrast to individual machine learning algorithms. Best comprehensive performance is observed in the ensemble "IB1+Random Forest+ J48+KStar" immediately second best result come from ensemble of machine learning algorithm "IB1+Random Forest+ J48+KStar+SimpleLogistic".

Fig. 6 gives the performance comparison based on percentage accuracy of the ensembles of machine learning algorithms used in proposed feature fusion based land usage identification method. Among the ensembles of machine learning algorithms examined the ensemble "IB1+Random Forest+ Simple Logistic +J48+KStar" have shown the overall best result where second best result is given by ensemble "Random Forest+ Kstar+IB1+J48". 


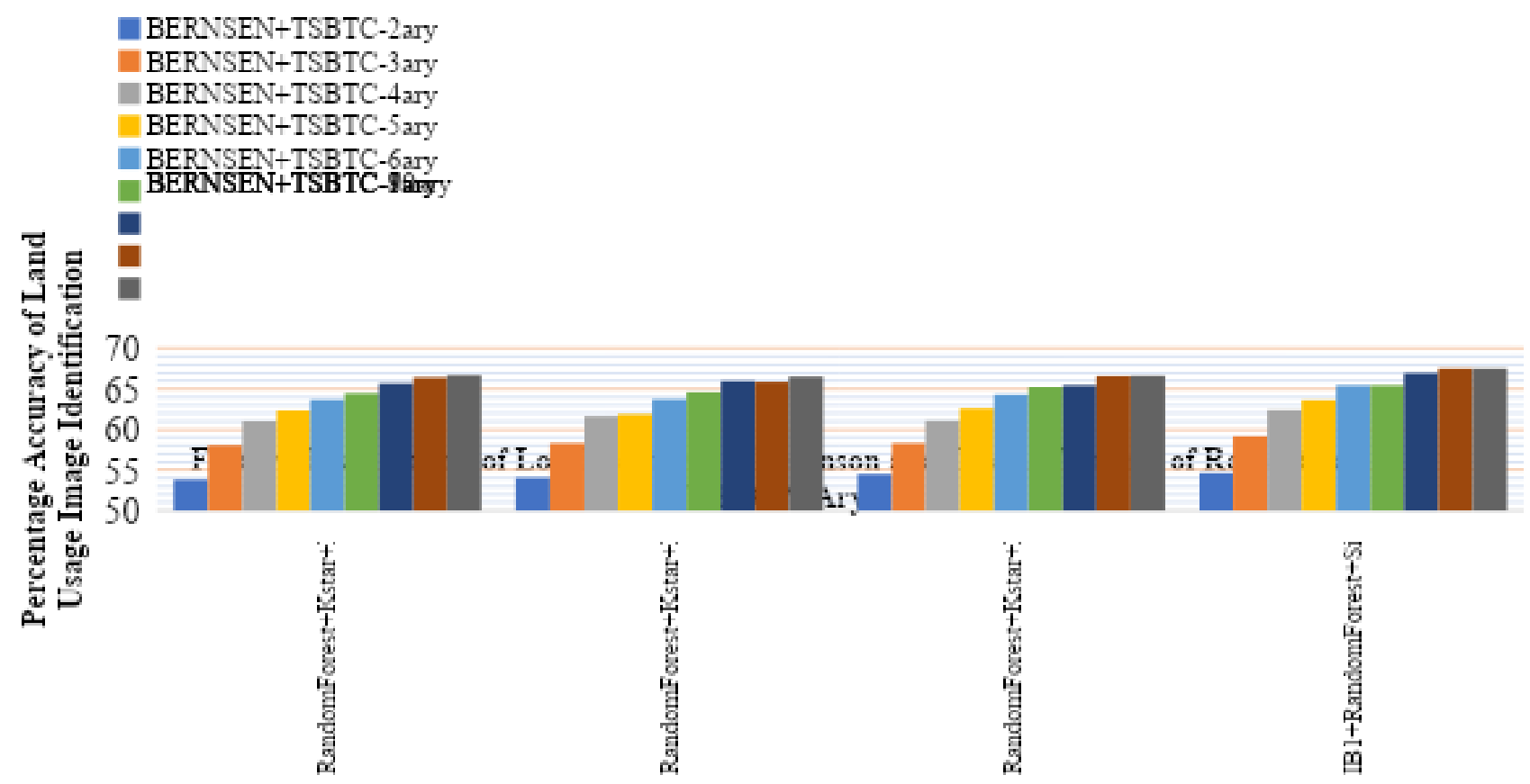

Fig. 7.Performance based on percentage Accuracy for feature level fusion combinations of local features of Bernsen and global features of respective TSBTC n-ary of examined ensembles of machine learning algorithms in proposed land usage identification technique.

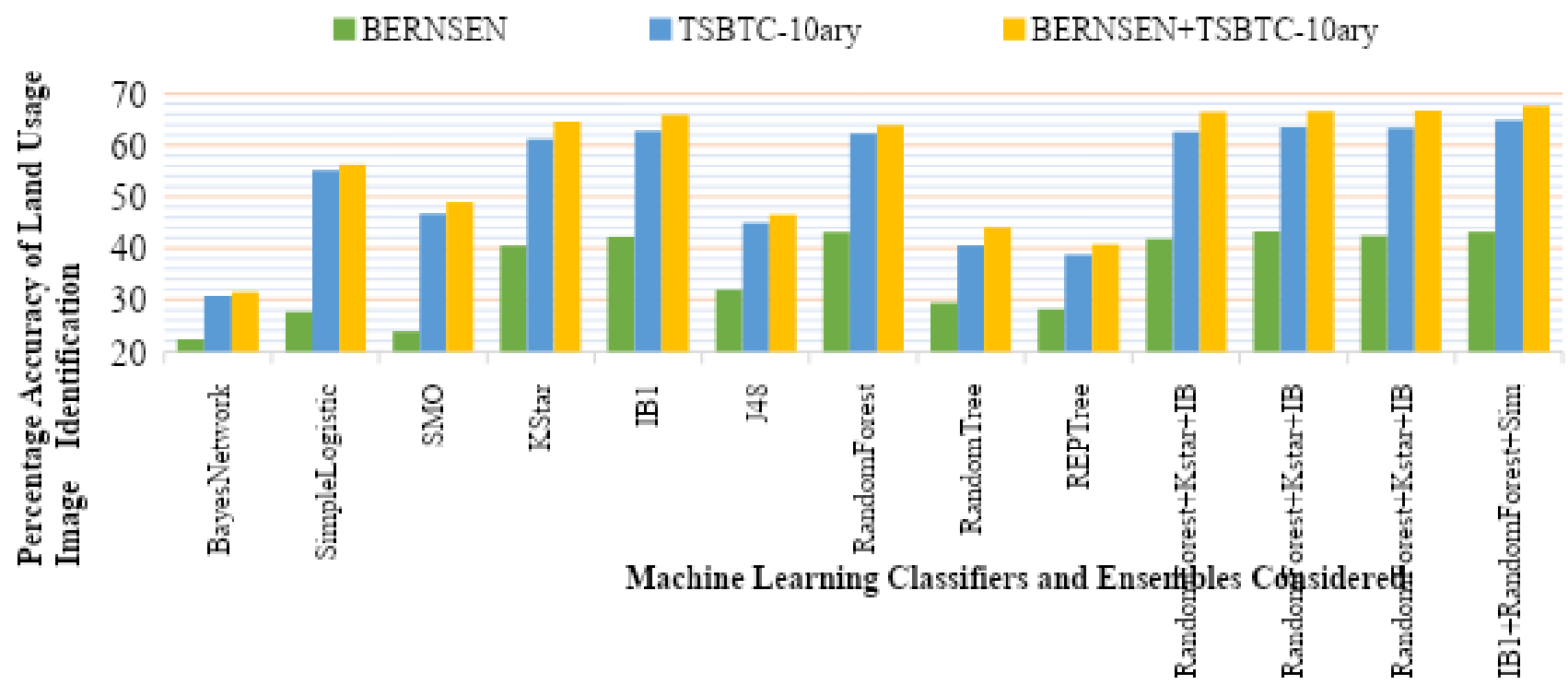

Fig. 8.Performance based on percentage Accuracy for variety of feature extraction methods examined as TSBTC 10ary, Bernsen and proposed feature level fusion combinations of Bernsen and TSBTC 10-ary for individual and respective examined ensembles of machine learning algorithms in proposed land usage identification technique.

Fig. 7 shows the comparison of percentage Accuracy for feature level fusion of local features of Bernsen and global features of respective TSBTC n-ary for ensembles of considered machine learning algorithms in proposed land usage identification technique. Here in all the considered ensembles, percentage accuracy is getting gradual increase with each upper level of TSBTC n-ary from TSBTC 2-ary till TSBTC 8-ary. From TSBTC 8-ary to TSBTC 10-ary, the marginal improvement is seen in considered ensembles whereas fusion of TSBTC 10-ary and Bernsen have shown the impressive performance across all the considered ensembles.

Fig. 8 represents the performance based on accuracy of considered feature extraction methods mainly global features with TSBTC 10-ary, local features with Bernsen and feature level fusion of TSBTC 10-ary and Bernsen applied for individual and ensemble of machine learning algorithms in proposed technique for land usage identification. 
As clearly inferred from the Fig. 8 the fusion of feature extraction

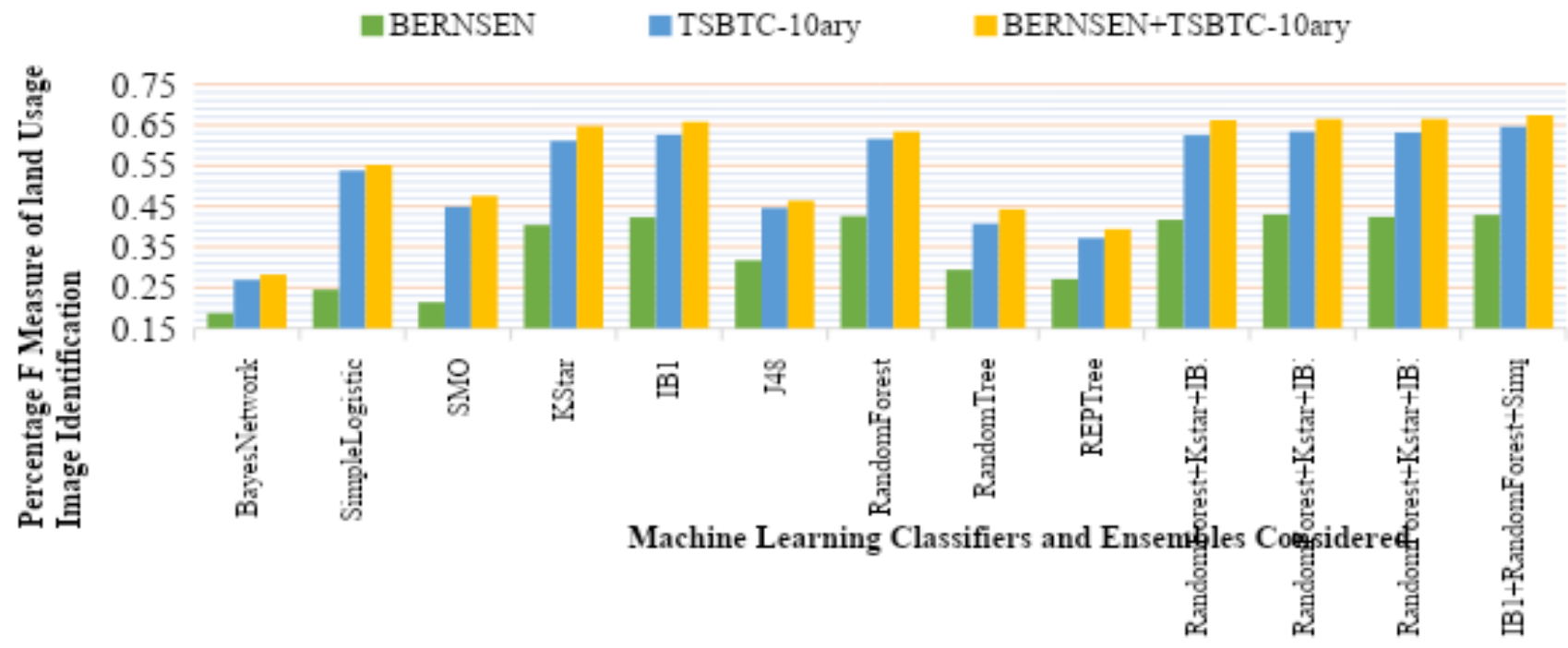

Fig. 9.Performance based on percentage F Measure for variety of feature extraction methods examined as TSBTC n-

ary, Bernsen and feature level fusion combinations of Bernsen and TSBTC n-ary for individual and respective

examined ensembles of machine learning algorithms in proposed land usage identification technique.

methods taking local and global features with "Bernsen + TSBTC 10-ary" applied for all considered machine learning algorithms have given the overall higher accuracy for land usage identification. Here the global features considered with TSBTC 10-ary have performed better than the local features considered with Bernsen thresholding. Also, the ensembles have the overall best percentage accuracy performance than individual machine learning algorithms.

Fig. 9 represents performance based on percentage $\mathrm{F}$ Measure for various feature extraction methods considered as global features with TSBTC n-ary, local features with Bernsen thresholding and fusion of TSBTC 10-ary and
Bernsen for particular considered individual machine learning algorithms and their ensembles in proposed land usage identification technique. Here for all the individual and ensemble of considered machine learning algorithms, the proposed fusion of TSBTC 10-ary and Bernsen have shown the better performance indicated as highest $\mathrm{F}$ Measure values. The global features undertaken with TSBTC 10-ary has better performance than local features considered with Bernsen feature extraction method. Overall the ensemble has the best performance $\mathrm{F}$ Measure values than the individual machine learning algorithms.

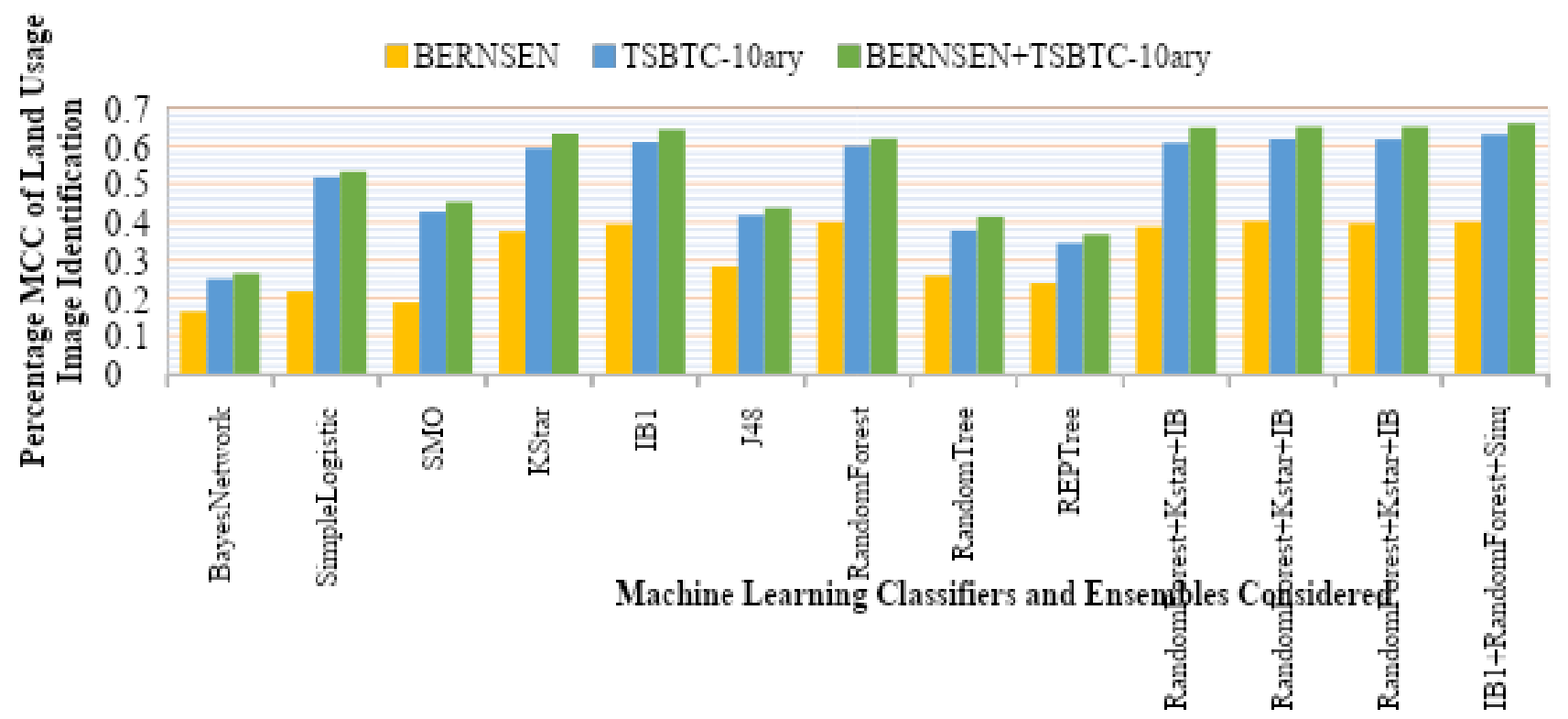

Fig. 10.Performance based on percentage Matthews Correlation Coefficient (MCC) for variety of feature extraction methods examined as TSBTC n-ary, Bernsen and feature level fusion combinations of Bernsen and TSBTC n-ary for individual and respective examined ensembles of machine learning algorithms in proposed land usage identification technique. 
In Fig. 10 Matthews Correlation Coefficient (MCC) based performance is measured for various feature extraction methods considered as global features with TSBTC 10-ary, local features with Bernsen thresholding and fusion of TSBTC 10-ary and Bernsen for particular considered individual machine learning algorithms and their ensembles. Here for all the individual and ensemble of considered machine learning algorithms fusion of TSBTC 10-ary and Bernsen have shown better performance indicated as higher MCC values. The global features undertaken with TSBTC 10-ary has superior performance than local features considered with Bernsen feature extraction method. Overall the ensemble has impressively outperformed well than the individual machine learning algorithm.

Overall the Land Usage Identification Accuracy, F Measure and MCC have shown that the proposed feature level fusion of global aerial image features extracted with TSBTC n-ary and local aerial image features extracted using Bernsen thresholding gives better performance for ensemble of classifiers proving the worth of the proposed technique.

\section{CONCLUSION}

The land usage identification in automated way using machine learning algorithms is interesting research dimension these days. The paper has attempted fusion of the global aerial image features extracted using TSBTC n-ary and local aerial image features extracted using Bernsen thresholding method. For improved land usage identification. The experimentation conducted on 2100 images of UC Merced Land Usage dataset of aerial images shown that proposed method gives better classification accuracy. Overall best performance is observed in fusion of Thepade Sorted Block Truncation Coding 10-ary and Bernsen thresholding features for ensemble of "IB1+Random Forest +Simple Logistic +J48+KStar". Also, the Matthews Correlation Coefficient (MCC) and F Measure values shown similar trend of improvements in results with proposed land usage identification method. Also paper proposed use of ensemble of machine learning classification over single classifier for land usage identification.

\section{REFERENCES}

1. Badre S. R. and Thepade S. D, "Novel Video Content Summarization Using Thepade's Sorted n-ary Block Truncation coding," Procedia Computer Science, vol. 79, December 2016, pp. 474-482.

2. Bernsen, J, "Dynamic thresholding of grey-level images," Eighth International Conference on Pattern Recognition. Proceedings, 27-31 October 1986, pp. 1251-1255.

3. Bulent Sankur and Mehmet Sezgin, "Survey over image thresholding techniques and quantitative performance evaluation," Journal of Electronic Imaging, vol. 13, January 2004, pp. 146-168.

4. Jinyi Zou, Wei $\mathrm{Li}$, Chen Chen and Qian $\mathrm{Du}$, "Scene classification using local and global features with collaborative representation fusion", Information Sciences, vol. 348, June 2016, pp. 209-226.

5. Khitem Amiri, Mohamed Farah and Ugur Murat Leloglu, "BoVSG: bag of visual SubGraphs for remote sensing scene classification," International Journal of Remote Sensing, vol. 41, no. 5, 2019, pp. 1986-2003.

6. Minakshi N. Vharkate and Vijaya B. Musande, "Retrieval of Remote Sensing Images Using Fused Color and Texture
Features with K-Means Clustering,” 2018 Fourth International Conference on Computing Communication Control and Automation (ICCUBEA), August 2018, pp. 1-6.

7. Rupali K.Bhondve, Sudeep D.Thepade and Rejo Mathews, "Performance Assessment of Color Spaces in Multimodal Biometric Identification with Iris and Palmprint using Thepade's Sorted Ternary Block Truncation Coding,", International Journal of Integrated Computer Applications and Research (IJICAR), July 2015.

8. Sudeep D. Thepade, Sanjay Sange, Rik Das and Suyash Luniya, "Enhanced Image Classification with Feature Level Fusion of Niblack Thresholding and Thepade's Sorted N-ary Block Truncation Coding using Ensemble of Machine Learning Algorithms," 2018 IEEE Punecon, November 2018, pp. 1-7.

9. Sudeep D. Thepade and Yugali Bafna, "Improving the Performance of Machine Learning Classifiers for Image Category Identification Using Feature Level Fusion of Otsu Segmentation Augmented with Thepade's N-Ary Sorted Block Truncation Coding," 2018 Fourth International Conference on Computing Communication Control and Automation (ICCUBEA), August 2018, pp. 1-6.

10. Thepade S. and Badre S., "Performance Comparison of Color spaces in Novel Video Content Summarization using Thepade's sorted n-ary Block Truncation Coding," International Conference \& Workshop on Electronics \& Telecommunication Engineering (ICWET 2016), February 2016, pp. 153-158.

11. Thepade S. and Das R., Ghosh S., "Novel Technique in Block Truncation Coding Based Feature Extraction for Content Based Image Identification," Lecture Notes in Computer Science, vol. 25, May 2015, pp. 55-76.

12. Yi Yang and Shawn Newsam, "Bag-Of-Visual-Words and Spatial Extensions for Land-Use Classification," ACM SIGSPATIAL International Conference on Advances in Geographic Information Systems (ACM GIS), January 2010, pp. 270-279.

13. Yansheng Li, Yongjun Zhang, Chao Tao, and $\mathrm{Hu}$ Zhu, "Content-Based High-Resolution Remote Sensing Image Retrieval via Unsupervised Feature Learning and Collaborative Affinity Metric Fusion,” Remote Sensing, vol. 8, August 2016.

14. Xinwei Zheng, Xian Sun, Kun Fu, and Hongqi Wang "Automatic Annotation of Satellite Images via Multifeature Joint Sparse Coding With Spatial Relation Constraint,", IEEE Geoscience and Remote Sensing Letters, vol. 10, no. 4, July 2013, pp. 652-656.

\section{AUTHORS PROFILE}

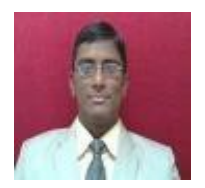

Dr.Sudeep D. Thepade is currently Professor in Computer Engineering Department at Pimpri Chinchwad College of Engineering affiliated to Savitribai Phule Pune University, Pune, Maharashtra, India. He has completed Ph.D. in 2011. He has more than 350 research papers to his credit published in International/ National Conferences and Journals. His domain of interest is Image Processing, Image Retrieval, Video Analysis, Video Visual Data Summarization, Biometrics and Biometric Liveness Detection. He is member of International Association of Engineers (IAENG) and International Association of Computer Science and Information Technology (IACSIT). He has served as Technical Program Committee member and Reviewer for Several International Conferences and Journals. 

Truncation Coding and Bernsen Thresholding with Ensemble Methods

Piyush R. Chaudhari is currently pursuing B. E. (Computer Engineering) from Pimpri Chinchwad College of Engineering, Savitribai Phule Pune University, Pune. His areas of interest is Problem Solving using various Data structure and Algorithms. He had completed his internship at OpenGenus Foundation at a position Algorithm and Data Structure Intern. Ranked $13^{\text {th }}$ highest Open Source Contributor all over the India in GirlScript Summer of Code (GSSOC'19). He is also head of Coding and Higher Studies Cells under PCCOE ACM Student Chapter. He had also hosted coding competition name "Codigo" in Anantya, He is the first student GitHub Campus Expert in Pune region.

Rik Das is currently Professor of Information Technology Department in Xavier Institute of Social Service, Ranchi, Jharkhand, India. He has completed Ph.D. in Information Technology from University of Calcutta. He has published 38 research papers, with 218 citations. His domain of interest is Machine Learning, Computer Vision, Deep Neural Networks, Image Processing and Image Classification. 